\title{
LOCAL EXISTENCE AND UNIQUENESS FOR EXTERIOR STATIC VACUUM EINSTEIN METRICS
}

\author{
MICHAEL T. ANDERSON
}

\begin{abstract}
We study solutions to the static vacuum Einstein equations on domains of the form $M \simeq \mathbb{R}^{n+1} \backslash B$ with prescribed Bartnik data $(\gamma, H)$ on the inner boundary $\partial M$. It is proved that for any smooth boundary data $(\gamma, H)$ close to standard round data on the unit sphere $\left(\gamma_{+1}, n\right)$, there exists a unique asymptotically flat solution of the static vacuum Einstein equations realizing the boundary data $(\gamma, H)$ which is close to the standard flat solution.
\end{abstract}

\section{INTRODUCTION}

In this short note, we prove a local existence and uniqueness theorem for solutions of the static vacuum Einstein equations, motivated by a well-known conjecture of Bartnik in general relativity. To describe the result and its setting, recall that the static vacuum Einstein equations on an $(n+1)$-dimensional manifold $M$ are given by

$$
u \text { Ric }=D^{2} u, \quad \Delta u=0,
$$

where $g$ is a Riemannian metric on $M$ and $u: M \rightarrow \mathbb{R}^{+}$is a positive (or non-negative) potential function. Here also Ric is the Ricci curvature of $g, D^{2}$ is the Hessian and $\Delta=\operatorname{tr} D^{2}$ is the Laplacian with respect to $g$. These equations are equivalent to the statement that the manifold $\mathcal{M}=S^{1} \times M$, with metric

$$
g_{\mathcal{M}}=u^{2} d \theta^{2}+g
$$

is Ricci-flat, i.e. $R i c_{g_{\mathcal{M}}}=0$, (the vacuum Einstein equations). In this work, we will only consider the situation where $M$ is diffeomorphic to the exterior of a ball $B \subset \mathbb{R}^{n+1}$, so $M \simeq \mathbb{R}^{n+1} \backslash B$. Moreover, we consider only solutions which are asymptotically flat (AF), in the sense that the metric $g$ and potential function $u$ satisfy

$$
g-g_{\text {Eucl }}=1+O\left(r^{n-1}\right), \quad u=1+O\left(r^{n-1}\right) .
$$

These conditions imply that the metric and potential in fact have an asymptotic expansion near infinity, cf. [5]; this will not be relevant here however.

Let $\mathbb{E}^{m, \alpha}$ be the space of all AF static vacuum solutions $(g, u)$ on $M$ which are $C^{m, \alpha}$ smooth up to the boundary $\partial M$. We assume $m \geq 2$ and $\alpha \in(0,1)$. This space is given the $C^{m, \alpha}$ topology with weight function $r^{n-1-\varepsilon}$. Let Diff ${ }_{1}^{m+1, \alpha}$ be the group of AF diffeomorphisms of $M$ which equal the identity on $\partial M$. This serves as the "gauge" group of the problem and the quotient space

$$
\mathcal{E}^{m, \alpha}=\mathbb{E}^{m, \alpha} / \operatorname{Diff}_{1}^{m+1, \alpha}
$$

is the corresponding moduli space of AF static vacuum solutions. It is proved in [1], cf. also [2], that $\mathcal{E}^{m, \alpha}$ is a smooth infinite dimensional Banach manifold; in particular the group Diff $1{ }^{m+1, \alpha}$ acts freely on $\mathbb{E}^{m, \alpha}$.

With each $(g, u) \in \mathbb{E}^{m, \alpha}$ one may associate its Bartnik boundary data $(\gamma, H)$, where $\gamma=g_{\partial M}$ is the induced metric on $\partial M$ and $H$ is the mean curvature with respect to the inward unit normal $N$

Date: October 30, 2018.

Partially supported by NSF grant DMS 1205947

MSC2010: 83C20, 58D29, 58J32. 
into $M$. Here the sign is chosen so that $H=n$ for the round unit sphere $S^{n}(1) \subset \mathbb{R}^{n+1}$. This choice of boundary data was introduced by Bartnik [3] in connection with his definition of quasi-local mass in general relativity. Note that the boundary data are invariant under the gauge group Diff ${ }_{1}^{m+1, \alpha}$.

One may thus define a Bartnik boundary map

$$
\begin{gathered}
\Pi_{B}: \mathcal{E}^{m, \alpha} \rightarrow M e t^{m, \alpha}(\partial M) \times C^{m-1, \alpha}(\partial M), \\
\Pi_{B}(g, u)=(\gamma, H) .
\end{gathered}
$$

This is a map of Banach manifolds, and again it is proved in [1], [2] that $\Pi_{B}$ is a smooth Fredholm map, of Fredholm index 0. This means that, at each $(g, u) \in \mathcal{E}^{m, \alpha}$, the derivative or linearization

$$
\begin{gathered}
D \Pi_{B}: T \mathcal{E}^{m, \alpha} \rightarrow T\left(M e t^{m, \alpha}\right)(\partial M) \times C^{m-1, \alpha}(\partial M), \\
D \Pi_{B}\left(h, u^{\prime}\right)=\left(h^{T}, H_{h}^{\prime}\right),
\end{gathered}
$$

is a Fredholm linear map, i.e. has finite dimensional kernel and cokernel, with $\operatorname{dim} \operatorname{Ker}\left(D \Pi_{B}\right)=$ $\operatorname{dimCoker}\left(D \Pi_{B}\right)$. Here $h$ is a symmetric bilinear form, $h^{T}$ is the restriction of $h$ to $T(\partial M)$ and $H_{h}^{\prime}$ is the variation of the mean curvature $H$ in the direction of the deformation $h$. The pair $\left(h, u^{\prime}\right)$ satisfy the linearization of the static vacuum equations (1.1) and so give an infinitesimal Einstein deformation.

The pure gauge infinitesimal deformations are of the form $\kappa=\left(\mathcal{L}_{Z} g, Z(u)\right)$, where $Z$ is a $C^{m+1, \alpha}$ vector field on $M$ with $Z=0$ on $\partial M$. These are of course "modded out" so don't appear in the finite dimensional kernel $K=\operatorname{Ker}\left(D \Pi_{B}\right)$.

A fundamental conjecture of Bartnik [3], [4], of importance in general relativity, is that the map $\Pi_{B}$ in (1.4) is a global diffeomorphism, i.e. one has global existence and uniqueness for this nonlinear boundary value problem, at least when the mean curvature $H>0$. As discussed in [2], this is not true in general and it remains a very interesting problem to understand the existence and uniqueness of solutions to the boundary value problem, i.e. the injectivity and range of the map $\Pi_{B}$. This issue is also closely related to a deeper understanding of the Bartnik quasi-local mass, cf. [3], [4].

Note that when the potential function satisfies $u=1$ on $M$, then $M$ is necessarily flat, so that in the context above, $(M, g) \subset\left(\mathbb{R}^{n+1}, g_{E u c l}\right)$ isometrically. We will call the solution with $u=1$, $g=g_{\text {Eucl }}$ and $M=M=\mathbb{R}^{n+1} \backslash B^{3}(1)$, the standard exterior solution; thus $M$ is the exterior of the round unit ball in $\mathbb{R}^{n+1}$ (up to isometry). The boundary data of the standard exterior solution are given by $(\gamma, H)$ with $\gamma=\gamma_{+1}=\gamma_{S^{n}(1)}$, and $H=n$.

Theorem 1.1. The standard exterior of the round unit ball $M=\mathbb{R}^{n+1} \backslash B^{3}(1), g=g_{E u c l}, u=1$ is a regular point of the boundary map $\Pi_{B}$, so that $D \Pi_{B}$ is an isomorphism. Thus

$$
K=\operatorname{KerD} \Pi_{B}=0
$$

and $D \Pi_{B}$ is surjective at the standard exterior solution.

In particular, if $\kappa=\left(k, u^{\prime}\right) \in T \mathbb{E}^{m, \alpha}$ is tangent to the standard exterior solution and $k^{T}=H_{k}^{\prime}=$ 0 , then $k=\delta^{*} Z$ with $Z=0$ on $\partial M=S^{n}(1)$.

A standard application of the inverse function theorem in Banach spaces gives the following:

Corollary 1.2. There is a neighborhood $\mathcal{U} \subset \operatorname{Met}^{m, \alpha}\left(S^{n}\right) \times C^{m, \alpha}\left(S^{n}\right)$ of the standard flat bounadry data $\left(\gamma_{+1}, n\right)$ such that for any $(\gamma, H) \in \mathcal{U}$, there is a unique solution $(M, g, u)$ of the static vacuum Einstein equations (1.1), up to isometry in Diff ${ }_{1}^{m+1, \alpha}$, for which

$$
\Pi_{B}(g, u)=(\gamma, H) .
$$


Corollary 1.2 generalizes a result of Miao [9], who proved this result when $\operatorname{dim} M=3$, for boundary data and static vacuum solutions which are invariant under reflection in the standard coordinate planes in $\mathbb{R}^{3}$, i.e. $\mathbb{Z}_{2} \times \mathbb{Z}_{2} \times \mathbb{Z}_{2}$ invariant. Note that this condition does not allow one imply that the standard exterior solution is a regular point of $\Pi_{B}$. This is discussed further in Remark 2.1. Theorem 1.1 gives the first example of a regular point of $\Pi_{B}$, with the exception of solutions near to the exterior of the horizon of the Schwarzschild metric discussed in [2].

I would like to thank Pengzi Miao for interesting discussions related to the topic of this paper.

\section{Proofs AND Remarks}

In this section we prove Theorem 1.1 and Corollary 1.2, and make several further remarks. The proofs are very simple, given the setting from the Introduction. To prove Theorem 1.1, it suffices to prove (1.6), since $D \Pi_{B}$ is Fredholm, of Fredholm index 0.

We begin with the Gauss equation or scalar constraint equation for the boundary $\partial M \subset M$. For a general Riemannian metric, this reads

$$
|A|^{2}-H^{2}+R_{\gamma}=R_{g}-2 \operatorname{Ric}(N, N),
$$

where $A$ is the 2nd fundamental form, $N$ is the unit normal pointing into $M$ and $R_{\gamma}, R_{g}$ are the scalar curvatures of $\gamma$ and $g$ respectively. For static solutions $R_{g}=0$ and $-\operatorname{Ric}(N, N)=$ $-u^{-1} N N(u)=u^{-1}\left(\Delta_{\gamma} u+H N(u)\right)$, where the second equality is a standard consequence of the relation $\Delta_{M} u=0$ in adapted (Fermi) coordinates about $\partial M$. (Here $\Delta_{\gamma}$ is the Laplacian on $(\partial M, \gamma))$. Hence one has

$$
u\left(|A|^{2}-H^{2}+R_{\gamma}\right)=2\left(\Delta_{\gamma} u+H N(u)\right),
$$

Consider the linearization of (2.1) in the direction of an infinitesimal static Einstein deformation $\kappa=\left(k^{\prime}, u^{\prime}\right) \in K=\operatorname{Ker} D \Pi$ about the standard round solution with $u=1$. For the standard round boundary data on $S^{n}(1) \subset \mathbb{R}^{n+1}$ one has $|A|^{2}=n, H^{2}=n^{2}$ and $R_{\gamma}=n(n-1)$, so that $|A|^{2}-H^{2}+R_{\gamma}=0$. We have $k^{T}=\left.k\right|_{\partial M}=0$ and $A=\gamma$ so that $\operatorname{tr} A_{k}^{\prime}=H_{k}^{\prime}=0$. It follows that

$$
2\left(\Delta_{\gamma} u^{\prime}+n N\left(u^{\prime}\right)\right)=2\left\langle A_{k}^{\prime}, A\right\rangle-2 H H_{k}^{\prime}=2 H_{k}^{\prime}-2 n H_{k}^{\prime}=0,
$$

on $\partial M$. Hence

$$
\Delta_{\gamma} u^{\prime}+n N\left(u^{\prime}\right)=0
$$

on $\partial M$.

Consider in general the equation

$$
\Delta_{\gamma} v+n N(v)=0
$$

on $\partial M$, where $v$ is harmonic on $\left(M, g_{E u c l}\right)$ with $v \rightarrow 0$ at infinity. Note that the variation $u^{\prime}$ satisfies these conditions, since

$$
0=(\Delta u)^{\prime}=\Delta^{\prime} u+\Delta u^{\prime}=\Delta u^{\prime}
$$

again since $u=1$ on $M$. Hence $u^{\prime}$ is harmonic on $M$. It is clear from (1.2) that $u^{\prime} \rightarrow 0$ at infinity in $M$.

We claim that the only solution to (2.3) is the trivial solution $v=0$. Thus, suppose first that $\max v>0$ at some point in $M$. Then $\max _{M} v>0$ occurs at a point $p \in \partial M$ and, by the Hopf maximum principle, cf. [8] for instance, $-N(v(p))>0$ where $-N$ is the outward unit normal to $M$. Hence $\Delta v(p) \leq 0$ and $N(v(p))<0$, contradicting (2.3). Hence $\max _{M} v=0$. The same argument working at a point achieving $\min v$ shows that $\min _{M} v=0$ so that $v=0$ which proves the claim. It follows in particular that $u^{\prime}=0$ on $M$.

By the linearized static vacuum equations, one has then $u^{\prime} R i c+u R i c^{\prime}=\left(D^{2}\right)^{\prime} u+D^{2} u^{\prime}$ which thus gives $\operatorname{Ric}_{k}^{\prime}=0$, so that $k$ is an infinitesimal flat deformation of ( $\left.M \backslash B^{n+1}(1), g_{E u c l}\right)$ with 
$k^{T}=0$. It is well-known that the round sphere is infinitesimally isometrically rigid in $\mathbb{R}^{n+1}$, i.e. the only infinitesimal isometric deformations of $S^{n}(1)$ are trivial, pure gauge deformations. The proof briefly is as follows. The Gauss-Codazzi equations for a hypersurface $\partial M$ in $\mathbb{R}^{n+1}$ state

$$
d A=0,
$$

where $d$ is the exterior covariant derivative on vector-valued 1-forms. The linearization of this in the direction $k$ above gives

$$
d A_{k}^{\prime}=0,
$$

so that $A_{k}^{\prime}$ is a Codazzi tensor, with vanishing trace. It follows by [6, Thm. 16.9] for instance that $A_{k}^{\prime}=0$ when $\partial M=S^{n}(1)$. Thus $k^{T}=A_{k}^{\prime}=0$. The linearized version of the fundamental theorem for hypersurfaces in $\mathbb{R}^{n+1}$ then implies that $\left(k, u^{\prime}\right)$ is a pure gauge deformation so that $\kappa=\left(k, u^{\prime}\right)$ equals zero in $K=\operatorname{Ker}(D \Pi)$. (Note here that Killing field deformations $X$ of $S^{n}(1)$ satisfy $\left.k=\delta^{*} X=0\right)$.

To prove Corollary 1.2, the inverse function theorem in Banach spaces implies that $\Pi$ is a local diffeomorphism near the standard exterior solution. Corollary 1.2 is just a restatement of this fact.

Another important class of static vacuum solutions are the Schwarzschild metrics of the form

$$
g_{m}=\left(1-\frac{2 m}{r^{n-1}}\right)^{-1} d r^{2}+r^{2} g_{S^{n}(1)},
$$

with $r>(2 m)^{1 /(n-1)}$ for $m \geq 0$ and $r>0$ for $m \leq 0$, and

$$
u=\sqrt{1-\frac{2 m}{r^{n-1}}} \text {. }
$$

Consider the exterior region $M=r^{-1}[1, \infty)$. One then has boundary data

$$
\gamma=\gamma_{+1}=g_{S^{n}(1)}, \text { and } H=n \sqrt{1-2 m} .
$$

The fact that regular points of the smooth map $\Pi_{B}$ are open implies that the exterior Schwarzschild domain $\left(M, g_{m}, u\right)$ above is a regular point of $\Pi_{B}$, for $m$ small. We conjecture that in fact all such round exterior Schwarzschild domains are regular points of $\Pi_{B}$. The proof of Theorem 1.1 however does not go through in this case, mainly since the relation (2.4) now becomes

$$
\Delta u^{\prime}=-\Delta^{\prime} u=\left\langle D^{2} u, k\right\rangle+\langle\beta(k), d u\rangle,
$$

where $\beta=\delta+\frac{1}{2} d t r$ is the Bianchi operator. It is not clear if the right side can be made to vanish, or have a particular sign, for $u$ of the form (2.6).

It is also unknown if general flat exterior solutions are regular points of $\Pi_{B}$, i.e $M=\mathbb{R}^{n+1} \backslash B$ with $B$ a ball with smooth boundary $\partial B=\partial M, g=g_{E u c l} u=1$. The main difficulty with the method above is handling the term $\left\langle A_{k}^{\prime}, A\right\rangle$. It would be very interesting to know this one way or the other.

We also conjecture that the standard flat exterior boundary data $\left(\gamma_{+1}, n\right)$, and similarly standard Schwarzschild exterior boundary data $\left(\gamma_{+1}, n \sqrt{1-2 m}\right)$ are uniquely realized by the flat and Schwarzschild metrics respectively. This is a global uniqueness conjecture, somewhat analogous to the well-known black hole uniqueness theorem for the Schwarzschild metric, cf. [7]. It does not appear that the methods used above can be adapted to settle this issue.

Remark 2.1. It is of interest to compare the proof of Corollary 1.2 with the main result of Miao in [9] which gives the same result in dimension 3 but with a $\mathbb{Z}_{2} \times \mathbb{Z}_{2} \times \mathbb{Z}_{2}$ symmetry condition imposed. Loosely speaking, and in physics terminology, Miao works off-shell and with a fixed gauge and boundary conditions, while the approach in this work is on-shell (i.e. on the space of 
solutions), without any gauge and studying the behavior of the gauge-invariant map to the boundary conditions. For this to work, it is important to know that the on-shell moduli space $\mathcal{E}^{m, \alpha}$ is itself a smooth Banach manifold.

In a little more detail, Miao proves that the linearized Einstein operator in a Bianchi gauge $D \Phi$, together with the the boundary conditions $(\gamma, H)$ imposed, has a surjective linearization at the standard round exterior in $\mathbb{R}^{3}$, when all data satisfy the symmetry condition $\mathbb{Z}_{2} \times \mathbb{Z}_{2} \times \mathbb{Z}_{2}$ above.

Note that the operator $T \sim D \Phi$ in [9] is not an isomorphism; the $L^{2}$ orthogonal complement of $\operatorname{ImT}$ is described in [9, Prop. 2]. In particular, it has non-zero gauge components $\eta$, $\hat{\eta}$. The proof of Theorem 1.1 above implies that $\operatorname{Ker} T=0$ so that the Fredholm operator $T$ has negative Fredholm index. An examination of the form of $(\operatorname{Im} T)^{\perp}$ given in $\left[9\right.$, Prop. 2] also shows that $D \Pi_{B}$ in (1.5) is surjective in dimension 3, [10] so that the two methods of proof are consistent.

\section{REFERENCES}

[1] M. Anderson, On boundary value problems for Einstein metrics, Geom. \& Topology, 12, (2008), 2009-2045.

[2] M. Anderson and M. Khuri, On the Bartnik extension problem for the static vacuum Einstein equations, Classical \& Quantum Gravity, 30, (2013), 125005.

[3] R. Bartnik, New definition of quasi-local mass, Phys. Rev. Lett., 62, (1989), 2346-2348.

[4] R. Bartnik, Mass and 3-metrics of non-negative scalar curvature, Proc. Int. Cong. Math., vol II, Beijing (2002), 231-240, Higher Ed. Press, Beijing, 2002.

[5] R. Beig and W. Simon, Proof of a multipole conjecture due to Geroch, Comm. Math. Phys., 78, (1980), 75-82.

[6] A. Besse, Einstein Manifolds, Springer Verlag, New York (1987).

[7] P. Chruściel, G. Galloway and D. Pollack, Mathematical general relativity: a sampler, Bull. Amer. Math. Soc., 47, (2010), 567-638.

[8] D. Gilbarg and N. Trudinger, Elliptic Partial Differential Equations of Second Order, $2^{\text {nd }}$ Edition, Springer Verlag, New York, (1983).

[9] P. Miao, On existence of static metric extensions in General Relativity, Comm. Math. Phys., 241, (2003), $27-46$.

[10] P. Miao, personal communication.

Dept. of Mathematics, Stony Brook University, Stony Brook, NY 11794-3651, USA

E-mail address: anderson@math.sunysb.edu

$U R L$ : http://www. math. sunysb.edu/ anderson 